\title{
COMPARISON OF TRAMADOL AND DEXMEDETOMIDINE FOR TREATMENT OF SHIVERING DURING SPINAL ANAESTHESIA IN CAESAREAN SECTION
}

\author{
Parthsarthi Prasad ${ }^{1}$
}

${ }^{1}$ Assistant Professor, Department of Anaesthesiology, GMC, Ambikapur, Surguja, Chhattisgarh, India.

ABSTRACT
BACKGROUND
Shivering is one of the most frequent complications of spinal anaesthesia, which is very distressing for the patients as well as
surgeons. Shivering increases metabolic rate, which causes increased oxygen consumption. Tramadol is opioid analgesic. It is
commonly used for treatment of shivering. Dexmedetomidine is alpha- 2 receptor agonist. It causes vasoconstriction by acting on
vascular smooth muscles and prevents shivering. In this study, efficacy of Tramadol and Dexmedetomidine for treatment of post
spinal shivering was compared.

\section{MATERIAL AND METHODS}

This study was prospective, double-blind, randomized controlled trial conducted in 50 patients of ASA grade I and II, ages between 18-40 years scheduled for caesarean section under spinal anaesthesia. Patients were divided in two groups of 25 each, receiving either Tramadol $0.5 \mathrm{mg} / \mathrm{kg}$ or Dexmedetomidine $0.5 \mu \mathrm{g} / \mathrm{kg}$ intravenously. Time of onset of shivering, time taken for cessation of shivering, recurrence and adverse effects were observed.

\section{RESULTS}

Both groups were demographically similar and comparable at baseline. Duration of surgery and onset of shivering were also similar in both groups. Dexmedetomidine taking significantly less time in treatment of shivering and having less recurrence rate in comparison to Tramadol. Nausea and vomiting were side effects of Tramadol only. Sedation was similar in both groups.

\section{CONCLUSION}

In conclusion both drugs are effective in treatment of shivering but Dexmedetomidine taking less time for cessation of shivering, less recurrence rate and having less side effects.

\section{KEY WORDS}

Spinal Anaesthesia, Shivering, Tramadol, Dexmedetomidine, Caesarean Section.

HOW TO CITE THIS ARTICLE: Prasad P. Comparison of tramadol and dexmedetomidine for treatment of shivering during spinal anaesthesia in caesarean section. J. Evolution Med. Dent. Sci. 2018;7(53):5601-5603, DOI: 10.14260/jemds/2018/1239

\section{BACKGROUND}

Most common anaesthetic technique for caesarean section is spinal anaesthesia. Shivering is one of the most common complication of spinal anaesthesia. ${ }^{1}$ It is very unpleasant to the patients and some patients find the accompanying cold sensation to be worse than the surgical pain. Shivering causes increase in metabolic rate, oxygen consumption and carbon di oxide production. It causes hypoxemia, lactic acidosis and raised intracranial pressure, which leads to tachycardia and hypertension.

Though the mechanism of origin of shivering is not clear, various hypotheses have been proposed to explain its occurrence. Perioperative hypothermia is the primary cause, which occurs due to neuraxial anaesthesia-induced inhibition of thermoregulatory mechanism. Shivering occurs as a thermoregulatory response to hypothermia or muscle activity with tonic or clonic patterns, and various frequencies have been noticed. ${ }^{2}$ Regional anaesthesia causes vasodilation, which leads to heat loss and decrease sympathetic tone.

'Financial or Other Competing Interest': None.

Submission 20-11-2018, Peer Review 14-12-2018,

Acceptance 20-12-2018, Published 31-12-2018.

Corresponding Author:

Parthsarthi Prasad,

Near Jaiswal Bhawan,

Bouripara, Ambikapur, Surguja,

Chhattisgarh-497001, India.

E-mail: parthsarthiprasad@yahoo.in

DOI: $10.14260 /$ jemds $/ 2018 / 1239$

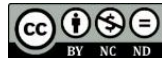

Both are responsible for shivering. ${ }^{3}$ Treatment of shivering are done by external heating and drugs. Commonly used drugs are Tramadol, Pethidine, Pentazocine, Clonidine, Dexmedetomidine etc.

Tramadol is most common drug used for treatment of shivering, 4 but having many adverse effects like nausea, vomiting dizziness etc. which causes discomfort to the patients. Dexmedetomidine is selective alpha- 2 receptor agonist, used as a sedative agent has better efficacy and less adverse effects as compared to Tramadol in various studies. ${ }^{5}$ Therefore, this comparative study was done to compare efficacy, haemodynamic responses and side effects of Tramadol and Dexmedetomidine for treatment of post spinal shivering.

\section{MATERIALS AND METHODS}

This study was prospective, double-blind, randomized controlled trial conducted in Govt. Medical college, Ambikapur, on 50 patients of ASA grade I and II, ages between 18 - 40 years scheduled for caesarean section under spinal anaesthesia. Patients sensitive to Tramadol or Dexmedetomidine, suffering from cardio - pulmonary, renal, endocrine and other major systemic diseases were excluded from the study.

\section{Sample Size Determination}

The sample size was estimated by difference in mean time required for cessation of shivering after giving the drugs in both groups. We assumed that a difference of 1 minute in 
mean time required for cessation of shivering would be clinically meaningful with expected standard deviation of 1.18 based on a preliminary study and at $95 \%$ confidence limit and $80 \%$ power, sample size of 23 was obtained in each group. With $10 \%$ non-response sample, 25 patients were included in the study in both groups.

Patients were randomly divided in two groups of 25 each, receiving either Tramadol $0.5 \mathrm{mg} / \mathrm{kg}$ or Dexmedetomidine $0.5 \mu \mathrm{g} / \mathrm{kg}$ intravenously after onset of shivering. Randomisation was done using computer-generated random number table. The double-blinding procedure was followed, in which the person administering the drug and the patients both were unaware as to which group the patient belonged to. The anaesthesiologist prepared drugs and coded them. The prepared drugs were given to resident, who was unaware of the contents, to be administered to the patients. At the end of the study results were analysed and then decoding done.

In the operation theatre multipara monitor were attached to the patient and pulse, NIBP, $\mathrm{SPO}_{2}$ and ECG were recorded. Preloading was done before spinal anaesthesia with $500 \mathrm{ml}$ ringer lactate. Under aseptic precautions spinal anaesthesia was administered at L3-4 space using $25 \mathrm{G}$ spinal needle with $0.5 \%$ heavy Bupivacaine. Patients were monitored by multipara monitor during surgery at regular intervals.

\section{Grading of shivering was done as follows:}

- Grade 0: No shivering

- Grade 1: One or more of the following: Piloerection, peripheral vasoconstriction, peripheral cyanosis without other cause, but without visible muscle activity

- Grade 2: Visible muscle activity confined to one muscle group

- Grade 3: Visible muscle activity in more than one muscle group

- Grade 4: Gross muscle activity involving the whole body.

Patients who developed either Grade 3 or Grade 4 of shivering were included in the study. Same criteria were used for grading of shivering during recurrence and patients with Grade 3 or 4 shivering were included.

If shivering was visible in the patient, then onset time of shivering was recorded and either of two drugs was given to the patient slowly after diluting in $5 \mathrm{ml}$ syringe. Time required for disappearance of shivering was recorded after giving drugs. Recurrence of shivering was also noted, and patients were given additional dose of Tramadol or Dexmedetomidine. Adverse effects like nausea, vomiting, hypotension, bradycardia and sedation were noted.

\section{Sedation Score was assessed with a Four-Point Scale as} per Filos 6

1. Awake and alert.

2. Drowsy, responsive to verbal stimuli.

3. Drowsy, arousable to physical stimuli.

4. Unarousable.

Bradycardia, hypotension and vomiting were treated with atropine, mephentermine and metoclopramide, respectively, in titrated doses when required.

\section{Statistical Analysis}

Statistical analysis was done by SPSS version 15 for analysing the collected data. Student's ' $t$ ' test was used for comparison of continuous data between two groups. Proportions were analysed by the use of the chi-square test or Fisher's test. Results were considered statistically significant if $\mathrm{p}<0.05$, highly significant if $\mathrm{p}<0.001$ and not significant if $\mathrm{p}>0.05$.

\section{RESULTS}

The results and observations of the cases in the present study are shown in the following tables and explained thereby

[A] Demographic Variables

\begin{tabular}{|c|c|c|c|}
\hline Parameter & Group- 1 & Group- 2 & P value \\
\hline Age (yrs) & $25.68 \pm 3.20$ & $24.72 \pm 3.40$ & $>0.05$ \\
\hline Weight (kg) & $53.28 \pm 4.62$ & $51.60 \pm 4.09$ & $>0.05$ \\
\hline $\begin{array}{c}\text { Duration of } \\
\text { surgery (min) }\end{array}$ & $45.80 \pm 4.93$ & $45.00 \pm 5.20$ & $>0.05$ \\
\hline
\end{tabular}

Table 1. Demographic Profile of Patients of Both Groups

Both groups were demographically similar and comparable. The differences in their means and standard deviations were statistically insignificant ( $p>0.05)$. Durations of surgeries in both groups were also similar and their differences were not statistically significant.

\section{[B] Observations for Shivering during Surgery}

\begin{tabular}{|c|c|c|c|}
\hline Parameter & Group- 1 & Group- 2 & P value \\
\hline Onset (min) & $19.96 \pm 5.52$ & $21.16 \pm 5.76$ & $>0.05$ \\
\hline $\begin{array}{c}\text { Cessation after } \\
\text { giving drug (min) }\end{array}$ & $5.12 \pm 1.27$ & $3.80 \pm 1.12$ & $<0.001$ \\
\hline Recurrence & 4 & 2 & $>0.05$ \\
\hline \multicolumn{4}{|l}{ Table 2. Parameters for Shivering } \\
\hline
\end{tabular}

The onset of shivering from starting of spinal anaesthesia were similar in both groups and their differences were not statistically significant. Time for cessation of shivering after giving Dexmedetomidine was significantly shorter than Tramadol $(p<0.001)$. Recurrence of shivering was less in Dexmedetomidine, but difference was not statistically significant $(p>0.05)$.

\section{[C] Adverse Effects}

\begin{tabular}{|c|c|c|c|}
\hline Adverse Effects & Group- 1 & Group- 2 & P value \\
\hline Nausea & 4 & 0 & $>0.05$ \\
\hline Vomiting & 3 & 0 & $>0.05$ \\
\hline Bradycardia & 0 & 0 & - \\
\hline Hypotension & 0 & 0 & - \\
\hline Sedation & 4 & 5 & $>0.05$ \\
\hline Resp. depression & 0 & 0 & - \\
\hline \multicolumn{2}{|c|}{ Table 3. Adverse Effects in Both Groups } \\
\hline
\end{tabular}

Nausea and vomiting were observed only in Tramadol group, but difference was not statistically significant ( $p>0.05$ ). Almost similar number of patients were sedated in both groups and difference was not statistically significant ( $p>0.05)$. There were no evidence of bradycardia, hypotension and respiratory depression in any patient of both groups. 


\section{DISCUSSION}

Most common anaesthesia for caesarean section is spinal anaesthesia. Shivering is very distressing experience for the patients during spinal anaesthesia. ${ }^{1}$ The exact mechanism of shivering during regional anaesthesia has not been fully established. The possible mechanisms include cessation of central thermoregulation, internal redistribution of body heat, and heat loss to the environment ${ }^{7}$. Vasoconstriction and shivering are restricted to the upper body during spinal anaesthesia, as they are inhibited below the level of blockade through sympathetic and somatic neural block. ${ }^{8}$

There are many non-pharmacological and pharmacological methods used to prevent heat loss and decrease shivering. Non-pharmacological methods include radiant heat warmers, warming the operation theatre, blankets, warm intravenous fluids and using anaesthetic drugs at body temperature. Various drugs like Tramadol, Pethidine, Pentazocine, Clonidine etc. used for treatment of post spinal anaesthesia shivering.

In this study, we studied the efficacy of dexmedetomidine in the treatment of post spinal anaesthesia shivering and compared its efficacy with tramadol in patients undergoing caesarean section. The onset of shivering from starting of spinal anaesthesia were similar in both groups and their differences were not statistically significant.

Tramadol is an opioid analgesic and commonly used for treatment of post spinal anaesthesia shivering.4 The antishivering action of Tramadol is probably mediated via its opioids or serotonergic and noradrenergic activity or both.

Dexmedetomidine is an alpha-2 adrenoreceptor agonist, used as sedative, analgesic, antihypertensive and antishivering agent. It reduces vasoconstriction and shivering threshold; thus, it acts on central thermoregulatory system. The role of dexmedetomidine in treatment of shivering has been evaluated in many studies. 5

The efficacy of dexmedetomidine is similar to a previous study done by Blaine Easley et al, ${ }^{9}$ who studied the role of dexmedetomidine in the treatment of postoperative shivering in children. All children had a cessation of shivering within $3.5 \pm 0.9 \mathrm{~min}$, while in our study cessation of shivering occurred in $3.8 \pm 1.12$ minutes.

In our study, the cessation of shivering with tramadol occurred in $5.12 \pm 1.27$ minutes. This is in accordance with previous literature, 10 in which cessation of shivering with tramadol occurred in $5.01 \pm 1.02$ minutes.

Although tramadol is an established drug for treatment of shivering, we found that dexmedetomidine is more effective than tramadol in treating post spinal anaesthesia shivering. We found that the time interval from the administration of treatment to cessation of shivering is significantly less with dexmedetomidine $(3.80 \pm 1.12$ minutes $)$ than with tramadol (5.12 \pm 1.27 minutes) $(\mathrm{P}<0.001)$.

In this study, the incidence of recurrence of shivering with dexmedetomidine was less $(2 / 25)$ as compared to tramadol $(4 / 25)$, but the difference was not statistically significant $(\mathrm{P}>0.05)$.

The side effects were found to be higher in the case of tramadol as compared to dexmedetomidine. In this study nausea and vomiting were observed only in Tramadol group. Although difference was not statistically significant ( $p>0.05)$, but nausea and vomiting are very unpleasant experience for the patient. There were no evidence of bradycardia or hypotension in any patient of both groups. Almost similar number of patients were sedated in both groups and difference was not statistically significant $(p>0.05)$. There was no incidence of respiratory depression and hypoxia in our study consequent to the loss of airway due to deeper planes of sedation. This sedation was found to be beneficial in the patients who were more comfortable with some amount of sedation from which they could be easily awoken.

The observations of the present study demonstrate that dexmedetomidine required less time for cessation of shivering, having less recurrence rate and less side effects.

\section{CONCLUSION}

Both tramadol and dexmedetomidine are effective in treatment of shivering but dexmedetomidine takes shorter time than tramadol for cessation of shivering and has less recurrence rate. The side effects of tramadol, like nausea and vomiting were not found with dexmedetomidine. So, we conclude that dexmedetomidine offers better thermodynamics than tramadol, with fewer side effects.

\section{REFERENCES}

[1] Crowley LJ, Buggy DJ. Shivering and neuraxial anesthesia. Reg Anesth Pain Med 2008;33(3):241-52.

[2] Daniel SI. Temperature monitoring. In: Millar RD, edr. Textbook of Anaesthesia. $5^{\text {th }}$ edn. New York: Churchill Livingstone Inc., 1994: p. 1367-89.

[3] De Witte J, Sessler DI. Perioperative shivering: Physiology and pharmacology. Anesthesiology 2002;96(2):467-84.

[4] Chaturvedi S, Domkondwar G. Control of shivering under regional anaesthesia using tramadol. Asian Arch Anaesthesiol Resusc 2002;57:491-6.

[5] Usta B, Gozdemir M, Demircioglu RI, et al. Dexmedetomidine for the prevention of shivering during spinal anesthesia. Clinics (Sao Paulo) 2011;66(7):1187-91.

[6] Filos KS, Goudas LC, Patroni O, et al. Hemodynamic and analgesic profile after intrathecal clonidine in humans. A dose-response study. Anesthesiology 1994;81(3):591-601.

[7] Glosten B, Sessler DI, Faure EA, et al. Central temperature changes are poorly perceived during epidural anesthesia. Anesthesiology 1992;77(1):10-6.

[8] Kurz A, Sessler DI, Schroeder M, et al. Thermoregulatory response thresholds during spinal anaesthesia. Anesth Analg 1993;77(4):721-6.

[9] Easley BR, Brady KM, Tobias JD. Dexmedetomidine for the treatment of postanesthesia shivering in children. Paediatr Anaesth 2007;17(4):341-6.

[10] Shukla U, Malhotra K, Prabhakar T. A comparative study of the effect of clonidine and tramadol on postspinal anaesthesia shivering. Indian $J$ Anaesth 2011;55(3):242-6. 